\title{
Psychological impact, coping strategies used and the effect of 'brief psychological intervention' on mental health of healthcare workers during the COVID-19 pandemic
}

\author{
Disha A. Vasavada ${ }^{1}$, Bhavesh R. Kanabar ${ }^{2}$, Renish B. Bhatt ${ }^{3}$, Deepak S. Tiwari ${ }^{4}$, \\ Lubna M. Nerli ${ }^{5}$ \\ ${ }^{1}$ Resident Doctor, Department of Psychiatry, M.P. Shah Medical College Jamnagar. \\ ${ }^{2}$ Tutor, Department of Preventive and Social Medicine, M.P. Shah Medical College, Jamnagar. \\ ${ }^{3}$ Senior Resident, Department of Psychiatry, M.P. Shah Medical College Jamnagar. \\ ${ }^{4}$ Professor and Head, Department of Psychiatry, M.P. Shah Medical College Jamnagar. \\ ${ }^{5}$ Resident Doctor, Department of Psychiatry, M.P. Shah Medical College Jamnagar.
}

Corresponding author: Disha Vasavada

Email - dishavasavada@gmail.com

\begin{abstract}
Background: Healthcare workers on the frontline who are directly involved in the treatment of COVID-19 patients are at a special risk of physical, psychological and social impact of the pandemic. This challenging situation is causing various mental health problems like distress, anxiety, depression, insomnia, and fear which affects their decision making ability in treating patients and also has long term physical and psychological implications. This study was aimed at investigating the psychological impact, coping strategies used and the effect of 'brief psychological intervention' on mental health of healthcare workers during the COVID-19 pandemic between May and October 2020.

Methodology: Total 175 healthcare workers participated in the interventional study. 'General health Questionnaire-12' and 'Impact of Events Scale-Revised (IES-R)' were used to assess psychiatric morbidity and posttraumatic stress respectively; 'brief-COPE' was used for assessing coping strategies used. An oral session of 'brief psychological intervention' was delivered and reassessment by IES-R was done 3 months later.

Results: Fear of spreading or contracting infection was more among females and nurses. Doctors had higher psychiatric morbidity and posttraumatic stress (PTS); there were no statistically significant gender differences in the same. Participants with higher psychiatric morbidity also had more PTS. Use of approach coping strategies showed least PTS. The interventional module was effective in stress alleviation.

Conclusion: COVID-19 pandemic had led to considerable stress among healthcare workers. Imparting regular psychological intervention can help in preparedness and building resilience to tackle the taxing circumstances that have arisen due to the pandemic.
\end{abstract}

Keywords: COVID-19, healthcare workers, psychological impact, intervention.

(Paper received $-12^{\text {th }}$ December 2020, Peer review completed $-16^{\text {th }}$ January 2021)

(Accepted $-20^{\text {th }}$ January 2020)

\section{INTRODUCTION}

COVID-19 pandemic is considered to be one of the greatest challenges the humankind has faced since the World War II, posing enormous health, social, environmental challenges to the human race and gravely disrupting the global economy [1]. Healthcare workers on the frontline who are directly involved in the diagnosis, treatment and care of patients with COVID-19 are at a special risk of physical, psychological and social impact of the pandemic as compared to the general population. Factors like the ever-increasing 
number of suspected and confirmed cases, overwhelming workload and manpower shortage, extensive media coverage, insufficient personal protective equipment (PPE), lack of a specific cure, confusion, fear of contagion and spreading infection to their family members, being placed in quarantine and isolation, lack of contact with family members, uncertainty and stigmatization are significant contributors to adverse impact on healthcare workers [2-4]. This challenging situation is causing a range of mental health problems like distress, anxiety, depression, insomnia, denial, anger and fear which could not only affect their attention, understanding and decision making ability in treating patients, but also have long term physical and psychological implications for themselves [5].

Studies among emergency room healthcare professionals in the past have observed clinical levels of PTSD ranging from $8-30 \%$ in the participants, which points to the lasting effects that pandemics can have on the mental health of healthcare workers [6-8]. The demand of extra vigilance in management of patients and taking care of their personal safety for an extended period of time, prolonged use of PPE and following stringent infection control procedures in order to avoid risking infection added to the psychical and psychological stress tremendously [9]. Moreover, accompanying dying patients and having to make end-oflife decisions for them when their kin cannot be by their side in periods of utmost uncertainty contributes to the traumatizing effect on the mental health of healthcare workers [10].

Among healthcare workers, specifically women, nurses that too with fewer years of work experience and frontline healthcare workers were found to have more severe symptoms of depression, anxiety, insomnia and distress [4]. Rising infection rates and mortality among healthcare workers is another factor responsible for increasing psychological stress [11].

Coping strategies have been classified into adaptive and maladaptive; emotion focused, problem focused and dysfunctional coping, approach and avoidant coping, etc [12]. Various studies in the past have found a negative association of adaptive coping strategies and a positive association of maladaptive coping strategies with rates of PTSD among participants [13-15]. A thorough knowledge about the preferred coping strategies can help a great deal in furnishing interventional techniques for healthcare workers.

Many interventional techniques like developing psychological interventional teams and peer support systems, partnering inexperienced workers with experienced ones, providing a place of rest, food and essential supply for the staff to isolate from their family, contact with family via video recordings, pre-job training to deal with psychological problems, regulations and directions for use of PPE, relaxation training and regular sessions with psychological counsellors for those in need of assistance have been tried regionally. Individually by institutes as well as regionally since the COVID-19 pandemic ensued [16-18].

Despite of this, adequate research into the psychological impact of COVID-19 pandemic on healthcare workers, particularly focusing on interventional strategies is lacking in India. Hence, to fill the gaps of previously conducted studies, our aim is to assess the psychological impact, coping strategies used and the effect of 'brief psychological intervention' on mental health of healthcare workers during the COVID-19 pandemic at a tertiary care hospital in Jamnagar, Gujarat.

\section{METHODOLOGY}

An interventional study was conducted over a period of 6 months, starting in May'2020 among healthcare workers employed in COVID-19 duty at a tertiary care hospital in Jamnagar, Gujarat after ethical approval from the Institutional Ethics Committee. Healthcare workers were contacted and were given a semistructured proforma which contained questions pertaining to demographic details, "General Health Questionnaire-12(GHQ-12)" and "Impact of Events Scale-Revised (IES-R)" to assess the psychological impact and "Brief-COPE" scale for evaluation of coping strategies used by the participants. Incomplete responses were exempted from the study.

Participants were then contacted in groups of 5-10 for a 20-30 minutes oral session of the 'Brief psychological intervention' module with adequate social distancing norms, which included general information regarding COVID-19, common psychological and physical reactions encountered during stress, positive coping strategies to adapt and negative strategies to avoid, specific techniques like ventilation, sleep hygiene, breathing exercises and ways to deal with stigma and discrimination. The module was prepared as per the guidelines of "Psychological first aid: guide for field workers" published by the joint collaboration of World 
Health Organization (WHO), 'War Trauma Foundation' and 'World Vision International' and "Psychosocial care in disaster management" furnished by the "National Institute of Disaster Management India' [19-20]. Participants were advised to follow the intervention on a daily basis and those who completed the session were then contacted after 3 months to assess the post-interventional effect by collecting their response for the 'IES-R'. Scales and the interventional module were translated to Gujarati and back translated to English by language experts.

\section{Materials}

General Health Questionnaire-12 (GHQ-12): The GHQ-12, a self-administered screening instrument is mainly used in differentiating patients suffering from psychiatric problems from those who are not mentally ill [21]. It consists of 12 items used to assess the extent of a mental problem over the past few weeks; scored using a Likert type scale (0-3) with scores ranging from 0 (substantial decrease in all symptoms) to 36 (substantial increase). The total score is calculated by summing the scores for each of the twelve items with $2 / 3$ points cut-off and the standardized Cronbach's alpha being 0.88 [22]. In the current study, participants having a GHQ-12 score of 1-12 were categorized as having 'no morbidity', 13-24 as 'mild morbidity' and 25-36 as 'moderate to severe morbidity'.

Impact of events scale - Revised (IES-R): The IES-R is the 22 items scale widely used as a self-report measure for traumatic stress. It also included six 'Hyperarousal' items to address the deficit in the previous IES (Impact of Events Scale) which only consisted of 'Intrusion' and 'Avoidance' subscales [23]. Rating is on a linear scale of 0 (not at all) to 4 (extremely) with respect to how distressing each item has been during the past week, with a score range of 0-88 [24]. IES-R score was categorized as: 0-8 $=$ 'No post-traumatic stress (PTS)', 9-25 = 'mild PTS', 26-43= 'moderate PTS' and 44-88 = 'severe PTS' according a recent study which used the same categorization [4]. The test-retest reliability across a six months interval was found to be ranging from 0.89 to 0.94 .

Brief-COPE scale: This scale designed to measure coping strategies has 14 subscales as follows, comprising of two items each: (a) acceptance (b) emotional support (c) humour (d) positive reframing (e) religion (f) active coping (g) instrumental support (h) planning (i) behavioural disengagement (j) denial (k) self distraction (1) self-blaming (m) substance use (n) venting [25]. Scoring is in the form of a likert scale ranging from 1 (I have not been doing this at all) to 4 (I have been doing this a lot). Cronbach's alpha value is considered adequate for the total scale, as all values exceed 0.60 [26].

\section{STATISTICAL ANALYSIS}

Data was collected from participants on printed questionnaire using pen and paper. Data entry and analysis was done using Microsoft excel and Epi-Info software. The socio-demographic profile was expressed in terms of frequency and percentage. Chi-square test was used for categorical data. The GHQ-12 and IES-R scores being non-normally distributed, were expressed as medians with interquartile ranges. The nonparametric Mann-Whitney U test and Kruskal-Wallis test were applied for comparison between two or more groups. Pearson correlation was used to find association between GHQ-12 and IES-R scores. WilcoxonSigned Ranks test was used to compare pre-intervention and post-intervention IES-R scores.

\section{RESULTS}

A total of 175 participants participated in the study; all 175 completed the pre-intervention proforma and 104 out of them completed the post-interventional proforma after attending the interventional session. Out of the total participants, $51.29 \%(n=102)$ were females and $41.71 \%$ were $(n=73)$ males. Occupation wise, $36 \%(n=63)$ were doctors, $40 \%(n=70)$ were nurses and $24 \%(n=42)$ were supportive staff participants. Majority (96\%) of the participants were followers of 'Hindu' religion and fell between the 21-30 years age group (57.14\%). Most of the participants were unmarried (53.14\%) at the time of study, had no children $(58.86 \%)$, and lived in hostel or as paying guest with friends (43.43\%). Most participants did not have any personal $(99.43 \%)$ or family history $(94.86 \%)$ of psychiatric illness or any major medical illness $(95.43 \%)$. 
Substance use was also denied by majority of the participants (90.29\%). 56.57\% (n=99) of the participants had a previous experience of working in the pandemic and $28 \%(n=49)$ affirmed for fear of getting infected. Higher proportion of nurses reported fear of getting infected or spreading infection to family members $(44.29 \%)$ as compared to doctors and supportive staff $\left(\chi^{2}=18.821, p<0.01\right)$; whereas gender wise, females were more fearful than males and both the findings were statistically significant $\left(\chi^{2}=12.706, p<0.01\right)$.

As per GHQ-12 scores, $14.8 \%(n=26)$ had mild and $1.7 \%(n=3)$ had moderate to severe psychiatric morbidity. $15.69 \%(\mathrm{n}=16)$ of the females had mild psychiatric morbidity while $13.70 \%(\mathrm{n}=10)$ males had mild and $4.11 \%(n=3)$ had moderate to severe psychiatric morbidity. Occupation wise, $19.05 \%(n=12)$ doctors, $18.57 \%(\mathrm{n}=13)$ nursing staff and $2.38 \%$ supportive staff $(\mathrm{n}=1)$ had mild psychiatric morbidity while only $4.76 \%(n=3)$ doctors had moderate to severe psychiatric morbidity.

Considering the IES-R scores, $38.10 \%(n=24)$ of the doctors, $35.71 \%(n=25)$ of the nursing staff and $30.95 \%$ $(n=13)$ of the supportive staff participants had posttraumatic stress (PTS). Gender wise, $36.65 \%(n=27)$ males and $34.31 \%(\mathrm{n}=35)$ females had PTS, without a statistically significant difference between both groups. Out of them, 30.14\% $(\mathrm{n}=22)$ males and $27.45 \%(\mathrm{n}=28)$ females had mild PTS; $4.11 \%(\mathrm{n}=3)$ males and $5.88 \%$ $(n=6)$ females had moderate PTS while $2.74 \%(n=2)$ males and $0.98 \%(n=1)$ females had severe PTS.

Table 1: Comparison of median GHQ-12 scores and median IES-R scores between different demographic variables $(\mathrm{N}=175)$.

\begin{tabular}{|c|c|c|c|c|c|c|c|c|}
\hline \multirow{3}{*}{\multicolumn{2}{|c|}{ Demographic variable }} & \multirow{4}{*}{\begin{tabular}{|c} 
No. \\
63 \\
\end{tabular}} & \multicolumn{3}{|c|}{ GHQ-12 } & \multicolumn{3}{|c|}{ IES-R } \\
\hline & & & \multirow{2}{*}{$\begin{array}{c}\text { Median } \\
\text { score (IQR) }\end{array}$} & \multicolumn{2}{|c|}{ Statistical value ${ }^{\#}$} & \multirow{2}{*}{$\begin{array}{c}\text { Median } \\
\text { score (IQR) }\end{array}$} & \multicolumn{2}{|c|}{ Statistical value } \\
\hline & & & & $H$ value & $p$ value & & $\mathbf{H}$ & p value \\
\hline \multirow{3}{*}{ Occupation } & Doctor & & $9.00(6.00)$ & \multirow{3}{*}{15.445} & \multirow{3}{*}{$<0.01$} & $5.00(15.00)$ & \multirow{3}{*}{0.592} & \multirow{3}{*}{0.744} \\
\hline & Nursing staff & 70 & $7.50(6.25)$ & & & $5.00(13.25)$ & & \\
\hline & $\begin{array}{l}\text { Supportive } \\
\text { staff }\end{array}$ & 42 & $6.00(2.00)$ & & & $5.50(8.00)$ & & \\
\hline \multirow{4}{*}{$\begin{array}{c}\text { Age group } \\
\text { (years) }\end{array}$} & $21-30$ & 100 & $8.00(6.00)$ & \multirow{4}{*}{9.903} & \multirow{4}{*}{0.019} & $5.00(13.00)$ & \multirow{4}{*}{1.392} & \multirow{4}{*}{0.707} \\
\hline & $31-40$ & 28 & $6.00(3.00)$ & & & $8.00(11.50)$ & & \\
\hline & $41-50$ & 31 & $6.00(5.00)$ & & & $4.00(9.00)$ & & \\
\hline & $>50$ & 16 & $6.00(1.75)$ & & & $3.5(14.00)$ & & \\
\hline \multirow{2}{*}{\multicolumn{2}{|c|}{ Demographic variable }} & \multirow{2}{*}{ No. } & Median & \multicolumn{2}{|c|}{ Statistical value $^{*}$} & Median & \multicolumn{2}{|c|}{ Statistical value $^{*}$} \\
\hline & & & score (IQR) & $Z$ value & $\mathrm{p}$ value & score (IQR) & $Z$ value & $\mathrm{p}$ value \\
\hline \multirow{2}{*}{ Gender } & Male & 73 & $7.00(4.50)$ & \multirow{2}{*}{-0.193} & \multirow{2}{*}{0.847} & $7.00(4.50)$ & \multirow{2}{*}{-0.329} & \multirow{2}{*}{0.847} \\
\hline & Female & 102 & $7.00(6.25)$ & & & $7.00(6.25)$ & & \\
\hline
\end{tabular}

\#test applied: Kruskal-Wallis test * test applied: Mann Whitney U test

Table 1 shows a statistically significant difference in median GHQ scores among participants according to their occupation and age group, but not gender. Whereas, there is no statistically significant difference in median IES-R scores as a function of occupation, age group or gender. GHQ-12 scores did not significantly differ as a function of place of posting $(\mathrm{z}=7.597, \mathrm{p}=0.108)$, living conditions $(\mathrm{z}=0.686, \mathrm{p}=0.710)$, marital $(\mathrm{z}=2.940, \mathrm{p}=0.230)$ and parenthood status $(\mathrm{z}=-1.540, \mathrm{p}=0.124)$, personal $(\mathrm{z}=-0.857, \mathrm{p}=0.392)$ or family history $(\mathrm{z}=-0.095, \mathrm{p}=0.924)$ of psychiatric illness, substance use $(\mathrm{z}=1.241, \mathrm{p}=0.538)$ and previous experience of working in a pandemic $(z=-0.144, p=0.149)$.
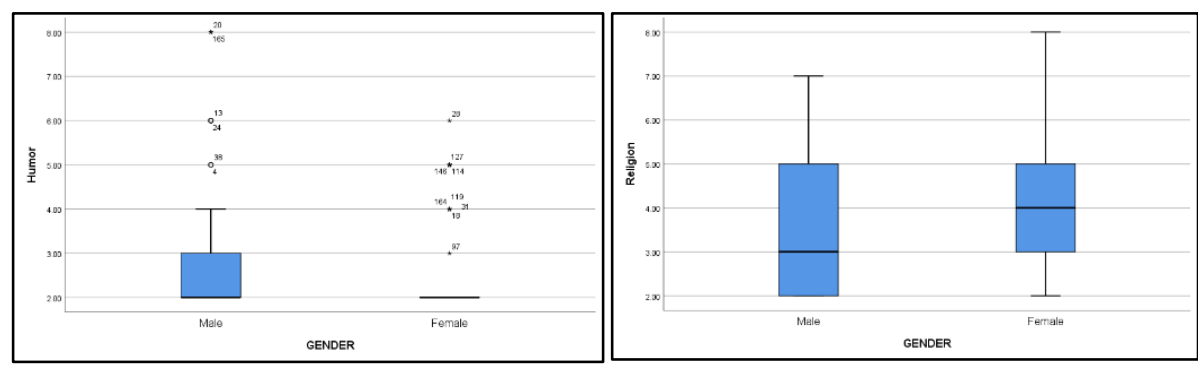

Figure 1: Box and whisker plot showing median scores of 'humour' and 'religion' coping strategies according to gender of participants. 
Figure 1 indicates that male participants have higher scores on 'humour' (Right skewed) as compared to females and females have higher scores on religion as compared to males which indicates greater use of 'humour' by males ( $\mathrm{z}=-2.122, \mathrm{p}=0.034)$ and 'religion' by females $(\mathrm{z}=2.271, \mathrm{p}=0.023)$ as a method of coping. 'Positive reframing' is also a preferred coping strategy among females. $(z=-1.986, p=0.047)$.
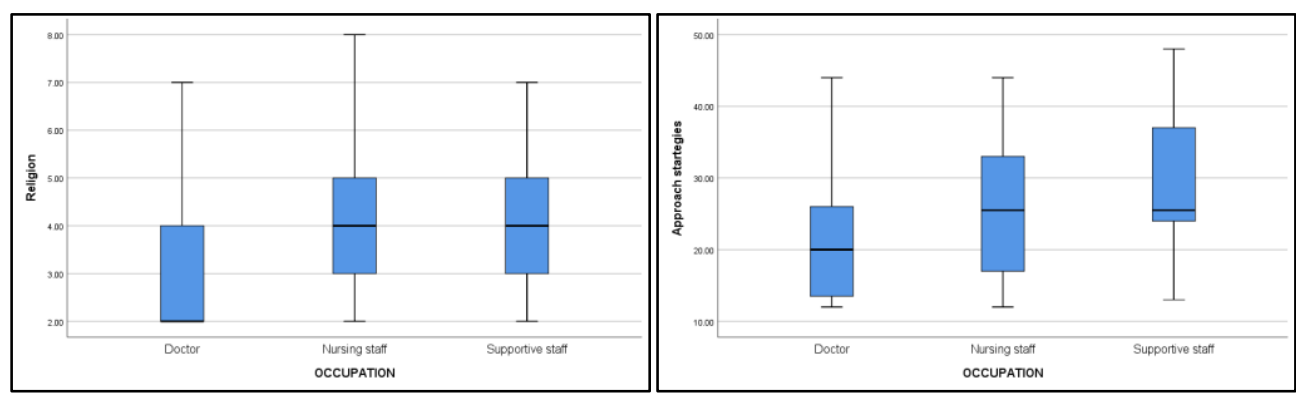

Figure 2: Box and whisker plot showing median scores of 'religion' and 'approach' coping strategies according to gender of participants.

Figure 2 indicates more use of 'religion' as a method of coping among nursing staff and supportive staff as compared to doctors, indicated by their higher median scores $(\mathrm{H}=21.564, \mathrm{p}<0.01)$. Higher median scores on 'approach' strategies' among nursing and supportive staff with more right skewing of the plot for supportive staff is suggestive of highest use of approach coping in the latter $(H=19.519, \mathrm{p}<0.01)$. Also, doctors prefer 'humour' as a coping strategy as compared to other groups $(\mathrm{H}=18.736, \mathrm{p}=<0.01)$. Moreover, all coping strategies are positively correlated with the total pre-intervention IES-R score, denoted by 'Pearson correlation test' $(\mathrm{p}<0.01)$.

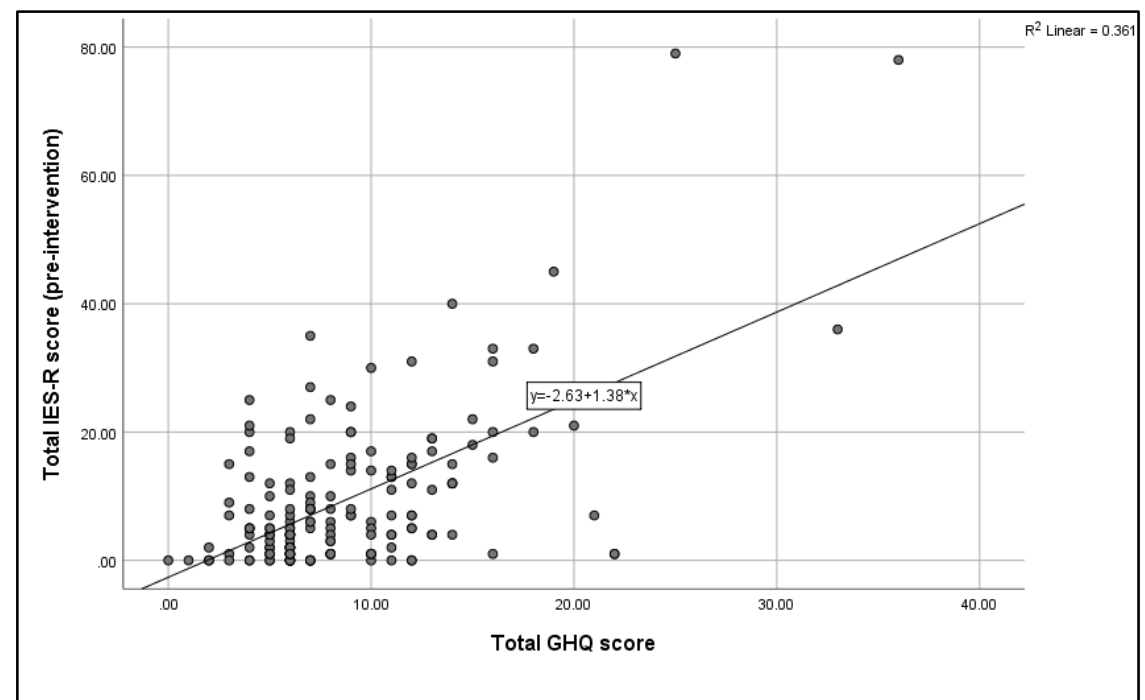

Figure 3: Correlation of total GHQ-12 score with total pre-intervention IES-R score.

Figure 3 shows a statistically significant positive correlation of Total IES-R (pre-intervention) score with Total GHQ-12 score by 'Pearson correlation test' with $\mathrm{r}$ value $=0.061$ and $\mathrm{p}<0.01$.

Table 2: Comparison between total pre-intervention and total post-intervention IES-R score $(\mathrm{N}=104)$.

\begin{tabular}{|c|c|c|c|}
\hline $\begin{array}{c}\text { Total IES-R score } \\
\text { (pre-intervention) }\end{array}$ & $\begin{array}{c}\text { Total IES-R score } \\
\text { (post-intervention) }\end{array}$ & \multicolumn{2}{|c|}{ Statistical value $^{\#}$} \\
\hline Median (IQR) & Median (IQR) & Z value & p value \\
\hline $5.00(11.00)$ & $4.00(6.00)$ & -2.388 & 0.017 \\
\hline
\end{tabular}


Table 2 indicates a statistically significant difference between pre-intervention and post-intervention total IES-R score $(\mathrm{z}=-2.388, \mathrm{p}=0.017)$.

\section{DISCUSSION}

COVID-19 pandemic has posed novel physical and psychological challenges for healthcare workers throughout the world. The fear of acquiring or spreading infection was significantly higher in females and nursing staff participants as compared to others. Bukhari and others reported higher worry of contracting the MERS-CoV among females [27] and Bettinsoli and others found that the fear of contracting infection was lower among hospital staff than doctors and nurses [28], both supporting the current study findings. Although, researchers established that concern of their family members' safety was a major stress inducing factor among healthcare workers which contrasts the current study findings [29].

The nature of their job and the unprecedented circumstances of the pandemic have put healthcare workers at a higher risk of psychiatric morbidity with $14.8 \%$ having mild and $1.7 \%$ of the participants having moderate to severe psychiatric morbidity. In contrast, $90 \%$ of the SARS survivor healthcare workers had a GHQ score above cut off, which is quite higher than the present condition; SARS survivor status being a probable reason for the former finding [3]. The current study shows mild psychiatric morbidity among females and doctors as compared to other gender and occupational groups. Two other studies similarly found that women had worse mental health as compared to males $[28,4]$. However, they did not find significant differences in mental health outcomes among doctors and nurses [28] while others reported a higher psychological burden among nurses compared to other healthcare workers [4,29]. Major involvement of doctors in making crucial life-saving decisions for the patients while nursing staff having the primary role of providing support to doctors by following the treatment orders could be reasons for higher psychological burden among them.

Participants belonging to 21-30 years age group had higher psychiatric morbidity as compared to other groups. However, place of posting, living conditions, marital and parenthood status, substance use, personal or family history of psychiatric illness and previous experience of working in a pandemic were not significantly related to psychiatric morbidity among participants. Researchers also found higher self-rated depression scores among younger staff ( $<30$ years), though not statistically significant [30]. Contrasting present study, researchers found higher psychiatric morbidity among healthcare workers posted in emergency and intensive care units and those working on frontline $[4,31]$. In another study, mental health of participants did not differ as per variables like marital status, parenthood status, living condition or the risk level of geographic area of residence [28]. Lesser resilience among younger staff and adequate training for proper use of PPE while catering to COVID-19 patients could be reasons for the findings of current study about psychiatric morbidity as a function of age and place of posting.

Studies conducted during the SARS and MERS-CoV outbreaks in the past have consistently pointed to a higher risk of 'Post Traumatic Stress Disorder' among healthcare workers [32]. In the present study, 28.57\% participants had mild post-traumatic stress (PTS), 5.14\% had moderate PTS and $1.71 \%$ had severe PTS.

Similar findings of a clinical concern of PTSD among $7.7 \%$ and $7.4 \%$ of the healthcare workers were reported in studies in Singapore and India respectively [33-34]. Females with moderate PTS were marginally more as compared to males, without a statistically significant difference. Overall, $38.10 \%$ doctors, $35.71 \%$ nursing staff and $30.95 \%$ supportive staff had PTS. Similarly, it was found that overall, being a woman and being a healthcare worker were cited as risk factors for adverse psychological outcomes like PTS [3]. Two recent studies also reported that occurrence of anxiety and PTS symptoms was higher in females, nurses and junior healthcare workers $[4,35]$. Whereas, some studies concluded that frontline healthcare workers were less prone to developing PTSS than second-line ones, possibly because the former were voluntarily selected and provided sufficient psychological preparation [36]. In contrast, Tan and others observed that nonmedically trained healthcare personnel had higher IES-R total and subscale scores as compared to medically trained healthcare workers [33]. Higher participants having mild PTS among supportive staff could be attributed to their less intensive infection control training, and overall less first-hand medical information on the outbreak which makes them more vulnerable to psychological impact of the pandemic. This emphasizes the importance of training for resilience building among healthcare workers who are at higher risk by virtue of their profession $[33,37]$. 
A positive correlation was found between the total GHQ-12 scores and IES-R scores of participants in the current study. Sim and others also found an association of high level of PTS symptoms with presence of psychiatric morbidity [38]. Yet another study reported higher depression, anxiety and PTS symptoms among healthcare worker SARS survivors with higher GHQ-12 scores, that evidently shows participants with higher psychiatric morbidity being prone to develop posttraumatic stress as compared to their psychologically healthier counterparts [3].

The post-interventional IES-R scores of all healthcare workers in the current study showed a statistically significant difference from the pre-interventional scores, suggesting effectiveness of the intervention. However, the 3 months duration between the pre and post-test could have led to psychological adaptation leading to lower scores. In contrast, two studies during past pandemics found higher rates of depression, anxiety, stress and posttraumatic stress symptoms among participants after a period of few months to a year $[3,39]$. Moreover, other comparative and interventional studies could not be found to corroborate the present findings.

Resorting to coping strategies to allay stress on an individual level is observed. In the present study, 'positive reframing' and 'religion' were preferred by females whereas 'humour' seen more among males as a way of coping. Another study exploring gender differences also found 'humour' being preferred by males [40]. 'Approach' coping strategies were preferably used more by supportive staff participants, while doctors preferred 'humour' and nurses preferred 'religion' as means of coping. In another study, it was found that significantly higher use of humour by physicians which resulted in lower posttraumatic stress [13]. Researchers have reported that more use of maladaptive coping strategies like avoidance, hostile confrontation and self-blame and less use of venting, humour and acceptance were associated with higher post-traumatic stress [14-15]. Overall, supportive staff group had the least participants with PTS symptoms, supporting the view that approach strategies are helpful in relieving the psychological stress. Similarly, studies in the past have found a negative correlation of active coping strategies and a positive correlation of passive coping strategies with depression, anxiety, stress and post-traumatic stress [41,42]. Positive correlation of approach coping strategies with post-traumatic stress in our study is still a contrasting finding, which points to the requirement of an active intervention beyond individual coping in order to alleviate the psychological impact of the pandemic among healthcare workers.

\section{CONCLUSIONS}

This study was aimed at investigating the psychological impact, coping strategies and the effect of 'brief psychological intervention' on mental health of healthcare workers during the COVID-19 pandemic. It was concluded that fear of getting infected or spreading it to others was higher among females and nursing staff participants. Younger participants (21-30 years) had higher psychiatric morbidity, although no significant gender differences in psychiatric morbidity and posttraumatic stress were found. Overall, doctors had the highest psychiatric morbidity and posttraumatic stress as compared to other occupational groups. Moreover, participants with higher psychiatric morbidity were prone to have higher posttraumatic stress. Use of approach coping strategies was the highest among supportive staff participants who also had the least PTS, suggesting a positive role in stress reduction. The interventional module was effective in alleviating the psychological stress among healthcare workers due to the unprecedented circumstances of the COVID-19 pandemic. Therefore, preparedness for foreseeable stressful circumstances among healthcare workers by imparting regular psychological interventions can play an important role in building resilience and have a positive effect on mental health and wellbeing of healthcare workers.

\section{Limitations and future scope:}

- A lack of control group in this study could be one of the confounding factors; hence future research that includes a control group could be conducted.

- There was only one follow up after the interventional session, therefore studies with more number of follow ups at regular intervals could strengthen the findings.

- The interventional module did not include any debriefing or other one-to-one brief therapeutic sessions. 


\section{REFERENCES}

1. Everyone Included: Social Impact of COVID-19 [Internet]. DISD. [cited 2020 Jul 17]. Available from: https://www.un.org/development/desa/dspd/everyone-included-covid-19.html

2. Bai Y, Lin CC, Lin CY, Chen JY, Chue CM, Chou P. Survey of Stress Reactions Among Health Care Workers Involved With the SARS Outbreak. Psychiatr Serv 2004;55(9):1055-7.

3. Lee AM, Wong JG, McAlonan GM, Cheung V, Cheung C, Sham PC, et al. Stress and Psychological Distress among SARS Survivors 1 Year after the Outbreak. Can J Psychiatry 2007;52(4):233-40.

4. Lai J, Wang Y, Cai Z, Hu J, Wei N, Wu J, et al. Factors Associated With Mental Health Outcomes Among Health Care Workers Exposed to Coronavirus Disease 2019. JAMA Network Open 2020;3:e203976.

5. Xiong Y, Peng L. Focusing on health-care providers' experiences in the COVID-19 crisis. Lancet Glob Health 2020;8(6):e740-1.

6. Adriaenssens J, de Gucht V, Maes S. The impact of traumatic events on emergency room nurses: findings from a questionnaire survey. Int J Nurs Stud 2012;49(11):1411-22.

7. Mills LD, Mills TJ. Symptoms of post-traumatic stress disorder among emergency medicine residents. J Emerg Med 2005;28(1):1-4.

8. Carmassi C, Gesi C, Simoncini M, Favilla L, Massimetti G, Olivieri MC, Conversano C, Santini M, Dell’Osso L. DSM-5 PTSD and posttraumatic stress spectrum in Italian emergency personnel: correlations with work and social adjustment. Neuropsychiatr Dis Treat 2016;12:375.

9. Chan AOM, Huak CY. Psychological impact of the 2003 severe acute respiratory syndrome outbreak on health care workers in a medium size regional general hospital in Singapore. Occup Med (Lond) 2004;54(3):190-6.

10. Braquehais MD, Vargas-Cáceres S, Gómez-Durán E, Nieva G, Valero S, Casas M, Bruguera E. The impact of the COVID-19 pandemic on the mental health of healthcare professionals. QJM: An International Journal of Medicine 2020;113(9):613-7.

11. Mahase E. Coronavirus: covid-19 has killed more people than SARS and MERS combined, despite lower case fatality rate. BMJ 2020;Feb 18:368.

12. García FE, Barraza-Peña CG, Wlodarczyk A, Alvear-Carrasco M, Reyes-Reyes A. Psychometric properties of the Brief-COPE for the evaluation of coping strategies in the Chilean population. Psicologia: Reflexão e Crítica 2018;31(1):22.

13. Dh P, Hk T, Ky T. Coping responses of emergency physicians and nurses to the 2003 severe acute respiratory syndrome outbreak. Acad Emerg Med 2005;12(4):322-8.

14. Maunder RG, Lancee WJ, Balderson KE, Bennett JP, Borgundvaag B, Evans S, Fernandes CM, Goldbloom DS, Gupta M, Hunter JJ, Hall LM. Long-term psychological and occupational effects of providing hospital healthcare during SARS outbreak. Emerg Infect Dis 2006;12(12):1924-32.

15. Sim K, Chong PN, Chan YH, Soon WSW. Severe acute respiratory syndrome-related psychiatric and posttraumatic morbidities and coping responses in medical staff within a primary health care setting in Singapore. J Clin Psychiatry 2004;65(8):1120-7.

16. Kang L, Li Y, Hu S, Chen M, Yang C, Yang BX, Wang Y, Hu J, Lai J, Ma X, Chen J. The mental health of medical workers in Wuhan, China dealing with the 2019 novel coronavirus. The Lancet Psychiatry 2020;7(3)e14.

17. Chen Q, Liang M, Li Y, Guo J, Fei D, Wang L, et al. Mental health care for medical staff in China during the COVID-19 outbreak. The Lancet Psychiatry 2020;7(4):e15-6.

18. World Health Organization. Mental Health Considerations during COVID-19 Outbreak. Geneva: World Health Organization;2020.

19. Snider L, Van Ommeren M, Schafer A. Psychological first aid: guide for field workers. Geneva, Switzerland: World Health Organization; 2011.

20. Sujata Satapathy. Psychosocial Care in Disaster Management : A Training of Trainers (ToT) module. NIDM, New Delhi. 2009.

21. Richard C, Lussier MT, Gagnon R, Lamarche L. GHQ-28 and cGHQ-28: implications of two scoring methods for the GHQ in a primary care setting. Soc Psychiatry Psychiatr Epidemiol 2004;39(3):235-43.

22. Petkovska MS, Bojadziev MI, Stefanovska VV. Reliability, Validity and Factor Structure of the 12-Item General Health Questionnaire among General Population. Open Access Maced J Med Sci 2015;3(3):478-83.

23. Creamer M, Bell R, Failla S. Psychometric properties of the Impact of Event Scale - Revised. Behav Res Ther 2003;41(12):1489-96.

24. Weiss DS. The Impact of Event Scale: Revised. In: Cross-cultural assessment of psychological trauma and PTSD. New York, NY, US: Springer Science + Business Media; 2007. p. 219-38.

25. Christianson S, Marren J. The impact of event scale--revised (IES-R). Medsurg Nurs 2012;21(5):321-3.

26. García FE, Barraza-Peña CG, Wlodarczyk A, Alvear-Carrasco M, Reyes-Reyes A. Psychometric properties of the Brief-COPE for the evaluation of coping strategies in the Chilean population. Psicologia: Reflexão e Crítica 2018;31(1):22.

27. Baumstarck K, Alessandrini M, Hamidou Z, Auquier P, Leroy T, Boyer L. Assessment of coping: a new french four-factor structure of the brief COPE inventory. Health Qual Life Outcomes 2017;15(1):8.

28. Bukhari EE, Temsah MH, Aleyadhy AA, Alrabiaa AA, Alhboob AA, Jamal AA, et al. Middle East respiratory syndrome coronavirus (MERS-CoV) outbreak perceptions of risk and stress evaluation in nurses. J Infect Dev Countries 2016;10(8):845-50. 
29. Bettinsoli ML, Di Riso D, Napier JL, Moretti L, Bettinsoli P, Delmedico M, et al. Mental Health Conditions of Italian Healthcare Professionals during the COVID-19 Disease Outbreak. Appl Psychol Health Well Being 2020;Oct 5.

30. Cai H, Tu B, Ma J, Chen L, Fu L, Jiang Y, et al. Psychological Impact and Coping Strategies of Frontline Medical Staff in Hunan Between January and March 2020 During the Outbreak of Coronavirus Disease 2019 (COVID-19) in Hubei, China. Med Sci Monit 2020;26:e924171-16.

31. Liang Y, Chen M, Zheng X, Liu J. Screening for Chinese medical staff mental health by SDS and SAS during the outbreak of COVID-19. J Psychosom Res. 2020;133:110102.

32. Naushad VA, Bierens JJ, Nishan KP, Firjeeth CP, Mohammad OH, Maliyakkal AM, et al. A Systematic Review of the Impact of Disaster on the Mental Health of Medical Responders. Prehosp Disaster Med 2019;34(6):632-43.

33. McAlonan GM, Lee AM, Cheung V, Cheung C, Tsang KW, Sham PC, et al. Immediate and Sustained Psychological Impact of an Emerging Infectious Disease Outbreak on Health Care Workers. Can J Psychiatry 2007;52(4):241-7.

34. Chew NWS, Lee GKH, Tan BYQ, Jing M, Goh Y, Ngiam NJH, et al. A multinational, multicentre study on the psychological outcomes and associated physical symptoms amongst healthcare workers during COVID-19 outbreak. Brain Behav Immun 2020;88:559-65.

35. Huang JZ, Han MF, Luo TD, Ren AK, Zhou XP. [Mental health survey of medical staff in a tertiary infectious disease hospital for COVID-19]. Zhonghua Lao Dong Wei Sheng Zhi Ye Bing Za Zhi 2020;38(3):192-5.

36. Li Z, Ge J, Yang M, Feng J, Qiao M, Jiang R, et al. Vicarious traumatization in the general public, members, and non-members of medical teams aiding in COVID-19 control. Brain Behav Immun 2020;88:916-9.

37. Styra R, Hawryluck L, Robinson S, Kasapinovic S, Fones C, Gold WL. Impact on health care workers employed in high-risk areas during the Toronto SARS outbreak. J Psychosom Res 2008;64(2):177-83.

38. Sim K, Huak Chan Y, Chong PN, Chua HC, Wen Soon S. Psychosocial and coping responses within the community health care setting towards a national outbreak of an infectious disease. J Psychosom Res 2010;68(2):195-202.

39. Lee SM, Kang WS, Cho A-R, Kim T, Park JK. Psychological impact of the 2015 MERS outbreak on hospital workers and quarantined hemodialysis patients. Compr Psychiatry 2018;87:123-7.

40. Vingerhoets AJ, Van Heck GL. Gender, coping and psychosomatic symptoms. Psychol Med 1990;20(1):12535 .

41. Si M-Y, Su X-Y, Jiang Y, Wang W-J, Gu X-F, Ma L, et al. Psychological impact of COVID-19 on medical care workers in China. Infect Dis Poverty 2020;9(1):113.

42. McPherson S, Hale R, Richardson P, Obholzer A. Stress and coping in accident and emergency senior house officers. Emerg Med J 2003;20(3):230-1.

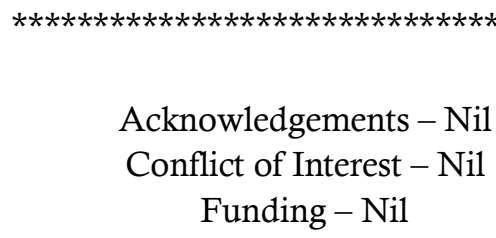

Indian Journal of Mental Health 2021; 8(2) 